\title{
SUMS OF INDEPENDENT RANDOM VARIABLES AND THE BURKHOLDER TRANSFORMS
}

\author{
J.-P. GABRIEL ${ }^{1}$
}

\begin{abstract}
This note shows a connection between the unconditionally a.e. convergence of series with independent increments and the a.e. convergence of their Burkholder transforms. Using this result, it is then proved that the $L_{1}$-bounded condition of Burkholder is the best one in the class of martingales, which assures the a.e. convergence of their transforms.
\end{abstract}

The purpose of this note is to indicate a connection between a certain type of convergence of sums with independent increments and the theory of the Burkholder transforms. The random variables (r.v.) will always be real and are defined on a probability space $(\Omega, \mathscr{F}, P)$. If $\left(Y_{n}\right)_{n \in N}$ is a family of r.v. then we denote by $S_{n}=\sum_{k=1}^{n} Y_{k}$ the partial sums of the series $\sum_{n=1}^{\infty} Y_{n}$. Let us suppose now that the $Y_{n}$ are independent. The most powerful theorem concerning the almost everywhere (a.e.) convergence of the series $\sum_{n=1}^{\infty} Y_{n}$ is certainly the "three series theorem" of Kolmogorov:

THEOREM 1. Let $\left(Y_{n}\right)_{n \in N}$ be a family of independent r.v. and let us define

$$
\bar{Y}_{n}= \begin{cases}Y_{n} & \text { if }\left|Y_{n}\right| \leqslant C, \\ 0 & \text { if }\left|Y_{n}\right|>C,\end{cases}
$$

where $C$ is a positive number. The series $\sum_{n=1}^{\infty} Y_{n}$ converges a.e. if and only if $\sum_{n=1}^{\infty} P\left\{Y_{n} \neq \bar{Y}_{n}\right\}, \sum_{n=1}^{\infty} E\left(\bar{Y}_{n}\right)$ and $\sum_{n=1}^{\infty} \operatorname{Var}\left(\bar{Y}_{n}\right)$ converge.

If, in this theorem, we replace $\sum_{n=1}^{\infty} E\left(\bar{Y}_{n}\right)$ by $\sum_{n=1}^{\infty}\left|E\left(\bar{Y}_{n}\right)\right|$, then we get a stronger result: The a.e. convergence of $\sum_{n=1}^{\infty} Y_{n}$ is now replaced by the a.e. convergence of $\sum_{k=1}^{\infty} Y_{n_{k}}$ for each rearrangement $\left(Y_{n_{k}}\right)_{k \in N}$ of $\left(Y_{n}\right)_{n \in N}$. In this case, we will say that $\sum_{n=1}^{\infty} Y_{n}$ is unconditionally a.e. convergent. We now recall the definition of the Burkholder transform. Let $\left(Y_{n}\right)_{n \in N}$ and $\left(V_{n}\right)_{n \in N}$ be two families of r.v. such that $\sup _{n \in N}\left|V_{n}\right| \leqslant 1$ (a.e.) and for each $n$ in $N, V_{n}$ is measurable with respect to the $\sigma$-algebra generated by $\left\{Y_{1}, Y_{2}, \ldots, Y_{n-1}\right\}$. The process $\left(T_{n}\right)_{n \in N}$, where $T_{n}=\sum_{k=1}^{n} V_{k} Y_{k}$, is called the (Burkholder) transform of $\left(S_{n}\right)_{n \in N}, S_{n}=\sum_{k=1}^{\infty} Y_{k}$, by the family of multipliers $\left(V_{n}\right)_{n \in N}$.

Received by the editors September 9, 1976 and, in revised form, January 13, 1977.

AMS (MOS) subject classifications (1970). Primary 60G45; Secondary 60G50.

Key words and phrases. Independent random variables, unconditionally a.e. convergence, Burkholder transform. martingales.

'Research supported by the Fonds National Suisse de la Recherche Scientifique. The results are parts of author's doctoral dissertation, directed by Professor R. Cairoli at the EPF-Lausanne. 
Burkholder proved that if $\left(S_{n}\right)_{n \in N}$ is an $L_{1}$-bounded martingale (i.e. $\left.\sup _{n \in N} E\left|S_{n}\right|<\infty\right)$, then each of its transforms is a.e. convergent. We now ask the following question: Is the condition $\sup _{n \in N} E\left|S_{n}\right|<\infty$ the optimal condition in the class of martingales which assures the a.e. convergence of their transforms? The answer is yes and we shall prove it for a simple subclass. We will now look at the transforms of a series with independent increments, because, when they are centered, the partial sums form a martingale.

TheOREM 2. Let $\left(Y_{n}\right)_{n \in N}$ be a family of independent r.v. The two following properties are equivalent:

(a) $\sum_{n=1}^{\infty} Y_{n}$ converges unconditionally a.e.;

(b) every Burkholder transform of $\sum_{n=1}^{\infty} Y_{n}$ is a.e. convergent.

Proof. We first prove that (b) implies (a). According to Theorem 1, $\sum_{n=1}^{\infty} P\left\{Y_{n} \neq \bar{Y}_{n}\right\}, \sum_{n=1}^{\infty} E\left(\bar{Y}_{n}\right)$ and $\sum_{n=1}^{\infty} \operatorname{Var}\left(\bar{Y}_{n}\right)$ converge. Let us choose a family of constant multipliers, $V_{n}=\operatorname{sign} E\left(\bar{Y}_{n}\right), n \in N$, and define

$$
\tilde{Y}_{n}= \begin{cases}V_{n} Y_{n} & \text { if }\left|V_{n} Y_{n}\right|=\left|Y_{n}\right| \leqslant C, \\ 0 & \text { if }\left|V_{n} Y_{n}\right|=\left|Y_{n}\right|>C,\end{cases}
$$

where $C$ is the number appearing in the definition of $\bar{Y}_{n}$. It is clear that $\tilde{Y}_{n}=V_{n} \bar{Y}_{n}$, and according to hypothesis (b) and Theorem 1,

$$
\sum_{n=1}^{\infty} E\left(\tilde{Y}_{n}\right)=\sum_{n=1}^{\infty}\left(\operatorname{sign} E\left(\bar{Y}_{n}\right)\right) E\left(\bar{Y}_{n}\right)=\sum_{n=1}^{\infty}\left|E\left(\bar{Y}_{n}\right)\right|
$$

converges. The proof is complete.

To prove that (a) implies (b), we first assume that the r.v. are symmetric. In this case, the r.v. $\bar{Y}_{n}$ are centered and the corresponding partial sums form a martingale. By Theorem 1, this martingale converges in quadratic mean and is then $L_{1}$-bounded. The Burkholder theorem completes the proof.

To do the general case, let us consider a family $\left(C_{n}\right)_{n \in N}$ of constant multipliers. We then have

$$
\begin{gathered}
\sum_{n=1}^{\infty} P\left\{C_{n} Y_{n} \neq C_{n} \bar{Y}_{n}\right\}=\sum_{n=1}^{\infty} P\left\{Y_{n} \neq \bar{Y}_{n}\right\}, \\
\sum_{n=1}^{\infty}\left|E\left(C_{n} \bar{Y}_{n}\right)\right| \leqslant \sum_{n=1}^{\infty}\left|E\left(\bar{Y}_{n}\right)\right| \text { and } \\
\sum_{n=1}^{\infty} \operatorname{Var}\left(C_{n} \bar{Y}_{n}\right) \leqslant \sum_{n=1}^{\infty} \operatorname{Var}\left(\bar{Y}_{n}\right),
\end{gathered}
$$

which implies the convergence of the three series in the left members. This shows that the theorem is also true when the multipliers are constant r.v.

Let us now introduce an independent copy $\left(Y_{n}^{\prime}\right)_{n \in N}$ of $\left(Y_{n}\right)_{n \in N}$. The r.v. $\left(Y_{n}-Y_{n}^{\prime}\right)_{n \in N}$ are symmetric and defined on $\left(\Omega \otimes \Omega^{\prime}, \mathscr{F} \otimes \mathscr{F}^{\prime}, P \otimes P^{\prime}\right)$, and the series $\sum_{n=1}^{\infty}\left(Y_{n}-Y_{n}^{\prime}\right)$ is unconditionally $P \otimes P^{\prime}$-a.e. convergent. Let $\left(V_{n}\right)_{n \in N}$ be a family of multipliers defined on $(\Omega, \mathscr{F}, P)$. We extend these r.v. 
to $\left(\Omega \otimes \Omega^{\prime}, \mathscr{F} \otimes \mathscr{F}^{\prime}, P \otimes P^{\prime}\right)$ in writing $V_{n}\left(\omega, \omega^{\prime}\right)=V_{n}(\omega)$, for each $\left(\omega, \omega^{\prime}\right)$ in $\Omega \otimes \Omega^{\prime}$, and it is clear that the new r.v. are still multipliers. The symmetry of $\left(Y_{n}-Y_{n}^{\prime}\right)_{n \in N}$ implies the $P \otimes P^{\prime}$-a.e. convergence of $\sum_{n=1}^{\infty} V_{n}\left(Y_{n}-Y_{n}^{\prime}\right)$. Furthermore, according to our previous result, for each $\omega$ in a set of probability 1 , the unconditional $P^{\prime}$-a.e. convergence of $\sum_{n=1}^{\infty} Y_{n}^{\prime}$ implies the $P^{\prime}$-a.e. convergence of $\sum_{n=1}^{\infty} V_{n} Y_{n}^{\prime}$. From these two facts, we conclude the $P$-a.e. convergence of $\sum_{n=1}^{\infty} V_{n} Y_{n}$.

We now return to the martingale problem with the following amusing theorem:

THEOREM 3. Let $\left(Y_{n}\right)_{n \in N}$ be a family of independent centered r.v., each taking at most two values. The unconditional a.e. convergence of $\sum_{n=1}^{\infty} Y_{n}$ is equivalent to $\sup _{n \in N} E\left(\left|\sum_{k=1}^{n} Y_{k}\right|\right)<\infty$.

Proof. According to Burkholder's theorem and Theorem 2, the sufficiency is obvious. Let us prove the necessity. If the r.v. are uniformly bounded, then it is well known that $\sum_{n=1}^{\infty} Y_{n}$ is convergent in quadratic mean and the result again follows from Burkholder's theorem and Theorem 2. We will then assume that we are not in this situation and we will write

$$
Y_{n}= \begin{cases}a_{n} & \text { with probability } p_{n}, \\ b_{n} & \text { with probability } 1-p_{n}\end{cases}
$$

Without loss of generality, we can assume that for each $n$ in $N,\left|a_{n}\right| \geqslant\left|b_{n}\right|$ and $0<p_{n}<1$. Let us define

$$
\bar{Y}_{n}= \begin{cases}Y_{n} & \text { if }\left|Y_{n}\right| \leqslant C \\ 0 & \text { if }\left|Y_{n}\right|>C\end{cases}
$$

where $C$ is a positive number, and consider the set $A=\left\{n \in N \mid P\left\{Y_{n}=\bar{Y}_{n}\right\}\right.$ $=1\}$, which according to our previous assumption is a proper subset of $N$. Theorem 1 implies the mean quadratic convergence of $\sum_{n \in A} Y_{n}$ and therefore $\sup _{n \in N} E\left(\left|\sum_{k \in A ; 1 \leqslant k \leqslant n} Y_{n}\right|\right)<\infty$. On the other hand, the inequality $\left|a_{n}\right| \geqslant$ $\left|b_{n}\right|$ implies that for each $n$ in $A^{c}$ :

$$
\bar{Y}_{n}= \begin{cases}0 & \text { with probability } p_{n}, \\ c_{n} & \text { with probability } 1-p_{n},\end{cases}
$$

where $c_{n}$ is either 0 or $b_{n}$. For each $n$ in the set $B=\left\{n \in A^{c} \mid c_{n}=0\right\}$, we have $P\left\{Y_{n} \neq \bar{Y}_{n}\right\}=1$ and, according to Theorem $1, \sum_{n=1}^{\infty} P\left\{Y_{n} \neq \bar{Y}_{n}\right\}<\infty$. The conclusion is that $B$ is finite, and therefore there exists $n_{0} \in N$ such that for each $n$ in $A^{c}$ and $n \geqslant n_{0}$, we have

$$
\bar{Y}_{n}= \begin{cases}0 & \text { with probability } p_{n}, \\ b_{n} & \text { with probability } 1-p_{n}\end{cases}
$$

The unconditional a.e. convergence of $\sum_{n=1}^{\infty} Y_{n}$ implies $\sum_{n=1}^{\infty}\left|E\left(\bar{Y}_{\underline{n}}\right)\right|<\infty$. Due to the fact that $E\left(Y_{n}\right)=0$ we have $\sum_{n=1}^{\infty}\left|E\left(\bar{Y}_{n}\right)\right|=\sum_{n \in A}\left|E\left(\bar{Y}_{n}\right)\right|$, and $a_{n}=b_{n}\left(\left(p_{n}-1\right) / p_{n}\right)$. Therefore 


$$
\sum_{\substack{n \in A^{c} \\ n>n_{0}}}\left|b_{n}\right|\left(1-p_{n}\right)=\sum_{n=n_{0}}^{\infty}\left|E\left(\bar{Y}_{n}\right)\right|<\infty,
$$

and from the equality $E\left|Y_{n}\right|=2\left|b_{n}\right|\left(1-p_{n}\right), \sum_{n \in A^{c} ; n>n_{0}} E\left|Y_{n}\right|<\infty$. By using the triangular inequality, we conclude that

$$
\begin{gathered}
\sup _{n \in N} E\left|\sum_{k=1}^{n} Y_{k}\right| \leqslant \sup _{n \in N} E\left(\left|\sum_{\substack{k \in A \\
1<k<n}} Y_{k}\right|\right)+E\left(\left|\sum_{\substack{k \in A^{c} \\
1<k<n_{0}}} Y_{k}\right|\right) \\
+\sup _{n \in N} E\left(\sum_{\substack{k \in A^{c} \\
n_{0}<k<n}}\left|Y_{k}\right|\right)<\infty .
\end{gathered}
$$

It is clear that the last theorem cannot be true for general r.v. The following example shows that it is wrong if the variables take three values.

EXAMPLE. Let $\left(Y_{n}\right)_{n \in N}$ be a family of independent centered r.v. such that $Y_{1}=0$ and

$$
Y_{n}= \begin{cases}1-n^{1+\alpha} & \text { with probability } 1 / 2 n^{1+\alpha}, \\ n^{1+\alpha} & \text { with probability } 1 / 2 n^{1+\alpha}, \\ 1 / 2\left(1-n^{1+\alpha}\right) & \text { with probability } 1-1 / n^{1+\alpha},\end{cases}
$$

for $n \geqslant 2$, where $\alpha$ is a positive number. Let us define

$$
\bar{Y}_{n}= \begin{cases}Y_{n} & \text { if }\left|Y_{n}\right| \leqslant \frac{1}{2} \\ 0 & \text { if }\left|Y_{n}\right|>\frac{1}{2}\end{cases}
$$

Then we have

$$
\begin{gathered}
\sum_{n=2}^{\infty} P\left\{Y_{n} \neq \bar{Y}_{n}\right\}=\sum_{n=2}^{\infty} \frac{1}{n^{1+\alpha}}<\infty, \\
\sum_{n=2}^{\infty}\left|E\left(\bar{Y}_{n}\right)\right|=\sum_{n=2}^{\infty} \frac{1}{2 n^{1+\alpha}}<\infty \text { and } \\
\sum_{n=2}^{\infty} \operatorname{Var}\left(\bar{Y}_{n}\right) \leqslant \sum_{n=2}^{\infty} \frac{1}{4\left(n^{1+\alpha}-1\right) n^{1+\alpha}}<\infty .
\end{gathered}
$$

The series $\sum_{n=1}^{\infty} Y_{n}$ is then unconditionally a.e. convergent. On the other hand, for each $n \geqslant 2, E\left|Y_{n}\right|=1$, and so the series does not converge in $L_{1}$. But the family $\left(\sum_{k=1}^{n} Y_{k}\right)_{n \in N}$ is a martingale with independent increments, and it is well known that in this case, $\sup _{n \in N} E\left(\left|\sum_{k=1}^{n} Y_{k}\right|\right)<\infty$ is equivalent to its convergence in $L_{1}$. We conclude that $\sup _{n \in N} E\left(\left|\sum_{k=1}^{n} Y_{k}\right|\right)=\infty$.

Conclusion. Theorem 3 shows that the $L_{1}$-bounded condition is the best one in the class of martingales which assures the a.e. convergence of their Burkholder transforms. On the other hand, the example shows that there exist non- $L_{1}$-bounded martingales whose transforms are a.e. convergent. 


\section{BIBLIOGRAPHY}

D. L. Burkholder, Martingale transforms, Ann. Math. Statist. 37 (1966), 1494-1504.

K. L. Chung, $A$ course in probability theory, Harcourt, Brace and World, New York, 1968.

J. L. Doob, Stochastic processes, Wiley, New York, 1953.

P. W. Millar, Martingales with independent increments, Ann. Math. Statist. 40 (1969), 1033-1041.

E. R. van Kampen, Infinite product measure and infinite convolutions, Amer. J. Math. 62 (1940). J.-P. Gabriel, Loi des grands nombres, séries et martingales indexées par un ensemble filtrant, Thèse de doctorat, EPF-Lausanne, Septembre 1975.

Courant Institute of Mathematical Sciences, New York University, New York, New YoRK 10012

Current address: Département de Mathématiques, École Polytechnique Fédérale de Lausanne, 61, avenue de Cour, CH-1007, Lausanne, Switzerland 